\title{
A chicken-and-egg problem: Which came first, the quantum state or spacetime?
}

\author{
Torsten Asselmeyer-Maluga \\ German Aerospace Center, Berlin, Germany
}

\begin{abstract}
In this essay I will discuss the question: Is spacetime quantized, as in quantum geometry, or is it possible to derive the quantization procedure from the structure of spacetime? All proposals of quantum gravity try to quantize spacetime or derive it as an emergent phenomenon. In this essay, all major approaches are analyzed to find an alternative to a discrete structure on spacetime or to the emergence of spacetime. Here I will present the idea that spacetime defines the quantum state by using new developments in the differential topology of 3- and 4-manifolds. In particular the plethora of exotic smoothness structures in dimension 4 could be the corner stone of quantum gravity.
\end{abstract}

\section{BASIC ASSUMPTIONS IN QUANTUM GRAVITY}

General relativity (GR) has changed our understanding of spacetime. In parallel, the appearance of quantum field theory (QFT) has modified our view of particles, fields and the measurement process. The usual approach for the unification of QFT and GR, to a quantum gravity, starts with a proposal to quantize GR and its underlying structure, spacetime. There is an unique opinion in the community about the relation between geometry and quantum theory: The geometry as used in GR is classical and should emerge from a quantum gravity in the limit (Planck's constant tends to zero). Most theories went a step further and try to get a spacetime from quantum theory. But what happens if this prerequisite is wrong? Is it possible to derive the quantization procedure from the structure of space and time? My own research program [1-13] seems to imply a simple answer: Yes. But in this essay I will discuss it from a more general perspective and analyze the basic assumptions of quantum gravity[22] first:

1. Spacetime has dimension 4 (also true in superstring theory after compactification).

2. Classical spacetime, i.e. the spacetime of GR or the spacetime as a limit of quantum gravity, is a smooth, non-compact 4-manifold with Lorentz structure and (trivial) codimension-1 foliation of the form $\Sigma \times \mathbb{R}(\Sigma$ a smooth 3-manifold, the space).

3. In the process of quantization, spacetime admits a discrete structure and one obtains the continuum only in the limit.

4. The quantum state in quantum gravity is realized as element (or vector) in some abstract state space (the space of all connections, the space of all spin networks etc.) with no direct reference to the classical spacetime.

5. Quantum gravity (as containing GR in some limit) must be background-independent, i.e. it does not explicitly depend on a concrete shape of the spacetime (diffeomorphism invariance).

6. Classical spacetime is induced (as emergent structure) by the quantum state in quantum gravity in the semiclassical limit.

All other explicit or implicit assumptions in concrete quantum gravity theories (like action principles or the dynamics) are very specific for the corresponding approach to quantum gravity. Let us analyze its relevance. The first assumption[23] is merely known from our experience but we must realize that four-dimensional spaces have an exceptional property among other spaces which is important for physics, exotic smoothness. The second assumption is motivated by GR and the research on the implementation of causality in GR. It is the starting point of Loop quantum gravity. The form of causality which was used to establish $\Sigma \times \mathbb{R}$ (see [14, 15]) is certainly too strong. There is a unique path into the past but not an unique path in the future, otherwise one needs the net of many-worlds. So, the second assumptions can be weakened to consider every kind of codimension- 1 foliation on a smooth, non-compact 4-manifold. It is known that a Lorentz structure will be a result of this foliation. The third assumption is the starting point of some approaches to quantum gravity (Causal nets, Regge calculus etc.) but all approaches are looking for discrete structures (spin networks and area quantization of Loop quantum gravity, finite string length in String theory) or to explicitly construct them (Dynamical triangulation etc.). Interestingly, all these approaches have problems in reaching the continuum limit. But is it really necessary to reduce the continuous spacetime to a discrete space? From GR we know that (by using diffeomorphism invariance) a point has no meaning in GR (see the next section for the whole discussion). But one can use relative techniques to relate two subspace of the spacetime to each other. Then only statements like "the two submanifolds intersect transversally" can be decided without introducing a concrete 
coordinate system. But statements of this kind are part of a mathematical theory called (differential) topology with discrete structures used to classify topological spaces. I will discuss these in more detail in section V. The fourth assumption is the most problematic one. Each approach to quantum gravity can be distinguished by its state space. So, the question must be: what is the "natural" state space of quantum gravity? Geometrodynamics started with the original superspace, i.e. the space of 3-metrics, with the Wheeler-deWitt equation as dynamics. This program was superseded by the introduction of Ashtekar variables (densitized coframe and $S U(2)$ connection) leading to Loop quantum gravity. The state space is the space of spin networks but the solution of the Hamilton constraint (as analog to the Wheeler-deWitt equation) is an unsolved problem. All state spaces are rather artificial and the relation to the geometry of the spacetime is poor. Part of the problem is the answer to the question: Is the quantum state real? If the answer is yes one has to consider quantum states in spacetimes. In particular one has to interpret the superposition of states. I will discuss this problem also in section V. The fifth assumption is induced directly from GR. It is one of the crucial points in GR: the theory has to be formulated without the reference to coordinates or the choice of a coordinate system does not influence the result. The implementation of this assumption is usually done by connecting it with the sixth assumption: there is no spacetime at the quantum level, the classical spacetime emerges from the deeper quantum level. Theories with a fuzzy spacetime including also noncommutative geometry are promising candidates for a direct implementation of quantum properties into spacetime.

In summary, especially the last four assumptions are questioned in this essay. In the following I will argue that the spacetime has the right properties for a spacetime-picture of QFT. Quantum gravity should be also part of this picture.

\section{THE FIRST SIGN: SPACETIME IS MORE THAN CLASSICAL}

When Einstein developed GR, his opinion about the importance of general covariance changed over the years. In 1914, he wrote a joint paper with Grossmann. There, he rejected general covariance by the now famous hole argument. But after a painful year, he again considered general covariance now with the insight that there is no meaning in referring to "the spacetime point A" or "the event A", without further specifications. Therefore the measurement of a point without a detailed specification of the whole measurement process is meaningless in GR. The reason is simply the diffeomorphism-invariance of GR which has tremendous consequences. Physical observables have to be diffeomorphism-invariant expressions. In most cases, this demand is impossible. The momentum of a moving particle is not diffeomorphism-invariant but it is physically meaningful. Therefore this momentum must be seen in the context of a measurement device which includes a concrete coordinate system. But generally, GR is background-independent of any coordinate system and, as usually thought, from the topology of the spacetime. So, if one fixes the topology then GR depends only on the (diffeomorphism-class) metric. But in section IV, I will discuss another possibility. Let $M$ be a smooth 4-manifold, say $M$ is the topologically $\mathbb{R}^{4}$ but with Lorentz structure, i.e. the Minkowski space. $M$ is flat and the GR vacuum equations are trivially fulfilled. The (smooth) atlas of $M$ is called the smoothness structure unique up to diffeomorphisms. One would expect that there is only one smooth atlas for $M$, all other possibilities can be transformed into each other by a diffeomorphism. But this is not true, see section IV. In fact, there are infinitely many non-equivalent smoothness structures on $M$ with no heuristic to distinguished one above the others as physically relevant.

\section{THE CONCEPT OF SPACETIME IN THE MAIN APPROACHES TO QUANTUM GRAVITY}

Quantum gravity as the unification of GR and QFT is currently an open problem but with a long list of possible candidates. Among them are Superstring theory and Loop quantum gravity. For each of these there are two classes: background-dependent and background-independent, respectively. But all proposals of a quantum gravity theory have one assumption in common: the spacetime has a foam-like structure or is discrete from the beginning. In string theory, the string has the extension of one Planck length and all structures below this length are unimportant. In particular the whole spacetime is seen as an emergent phenomenon which must be derived from the full theory. The background-dependence is a problem but it will be resolved in the conjectured M-theory. The relation to GR is interesting. At the non-quantized level, the world surface of the string is embedded in some background. If this embedding is fixed then the curvature of the world surface is determined by the curvature of the embedding space and vice versa. On the quantum level, one usually argues with the help of the $\beta$ function, encoding the dependence of a coupling parameter in the renormalization group. For a conformal invariant theory (conformal invariance of the world surface), this function has to vanish. In the case of string theory, one obtains the vanishing of the scalar curvature of the embedding space or the Einstein equation for the vacuum. In Loop quantum gravity, the central theme is background-independence installed by the vanishing of three constraints. One of the main results of the 
theory is the quantization of the area and the volume. It seems that spacetime must have a discrete structure. But the result was obtained by using an eigenvalue equation. As in the case of a harmonic oscillator, the underlying space is continuous but the spectrum of the area or volume operator is discrete. In section V, I will give an example of a class of spaces (hyperbolic 3-manifolds) with this property. Many other proposals start with a discrete structure from the scratch: Causal Sets, Quantum Causal Histories, Dynamical Triangulations etc. Therefore in all current quantum gravity proposals, the spacetime has a discrete structure or emerges from a discrete structure. But is the spacetime model of a smooth manifold dead? Mathematicians have found many interesting properties of 3- and 4-manifolds.

\section{THE SECRET REVOLUTION: OUR UNDERSTANDING OF 3- AND 4-MANIFOLDS}

According to GR, spacetime is a smooth 4-manifold carrying a Lorentz-structure. The existence of a Lorentzstructure is closely related to the existence of a codimension- 1 foliation. Therefore one also has to consider the spatial component, a smooth 3-manifold, as leaf of the foliation. From the mathematical point of view, one has to look into the theory of 3- and 4-manifolds (see [4] for more details and the references).

The central concept is the smooth manifold as a generalization of a surface. Riemann was the first one to do extensive work generalizing the idea of a surface to higher dimensions. The name manifold comes from Riemann's original German term, Mannigfaltigkeit. In his Göttingen inaugural lecture ("Ueber die Hypothesen, welche der Geometrie zu Grunde liegen"), Riemann described the set of all possible values of a variable with certain constraints as a Mannigfaltigkeit, because the variable can have many values. By induction, Riemann was able to define an $n$-times extended manifold. Then Poincare gave a definition of a (differentiable) manifold (variété) as subset of some Euclidean space which served as a precursor to the modern concept of a manifold. During the 1930s Hassler Whitney and others clarified the foundational aspects of the subject. In particular, a manifold was defined intrinsically with charts and transition functions and the equivalence of this definition with Poincare's Euclidean subsets was shown (Whitney's embedding theorem). The development of algebraic topology gave the right frame to investigate the manifolds (classification of surfaces by homology, the fundamental group etc.). The first surprise came in 1957. John Milnor constructed the first seven exotic 7-spheres (7-dimensional spheres which are homeomorphic but not diffeomorphic to each other). Therefore there must be a difference between a smooth manifold (all charts as maps to a Euclidean space and transition functions are smooth maps) and a topological manifold (the maps are now only continuous). The new topic "differential topology" was born. The (smooth) atlas (all smooth charts and transitions function to cover the manifold) was called smoothness structure. But how many in-equivalent smoothness structures on a $n$-manifold exists? Using powerful methods (like the h-cobordism theorem), Kervaire and Milnor were able to determine it for the spheres. The following table gives the values up to dimension 20 :

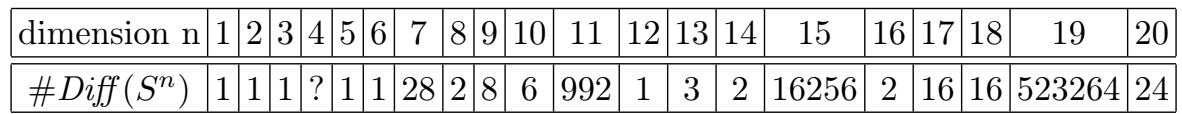

In particular, it was shown that the number of in-equivalent smoothness structure on a $n$-manifold is only finite for $n>4$. The uniqueness in low dimensions $n<4$ is a classical result. Therefore only the 4 -dimensional case was open. All methods developed for 2- and 3-manifolds or for higher-dimensional $(n>4)$ manifolds were useless in this dimension. In 1973, Andrew Casson gave lectures about a new construction in dimension 4, which he called flexible handles (now known as Casson handles). The mimeographed notes of these three lecture came to Micheal Freedman and occupied him over the next 7 years. Eventually, he successfully classified simply-connected, compact 4-manifolds. Meanwhile, Simon Donaldson (a student of M. Atiyah) started the investigation of anti-self-dual connections of a $S U(2)$ gauge theory, in physics known as instantons. As a surprising result, he proved that not all topological, compact 4-manifolds are smoothable. With this result in mind, Kirby, Freedman and Gompf constructed the first examples of an exotic $\mathbb{R}^{4}$, i.e. a space homeomorphic to $\mathbb{R}^{4}$ but not diffeomorphic. The second surprise came in the form of the number of inequivalent smoothness structures: there are countably infinite many for most compact 4-manifolds and uncountably infinite many for most non-compact 4-manifolds including $\mathbb{R}^{4}$. The development of this topic is not complete. In particular for the simplest compact 4-manifold, the 4-sphere, we do not know the number of in-equivalent smoothness structures. Most mathematicians conjecture that there are countably infinite many structures on the 4 -sphere. So, dimension 4 is exceptional for smoothness!

One point is also important for quantum gravity. With Milnor's discovery of exotic 7-spheres, one started to look for the existence of other structures on manifolds. In particular the existence question for a triangulation (or piecewise-linear structure, PL-structure) was very interesting. The surprising result of Cerf for manifolds of dimension smaller than 7 was simple: PL-structure (or triangulations) and smoothness structure are the same. This implies that every PL-structure can be smoothed to a smoothness structure and vice verse. Therefore the discrete approach (via triangulations) and the smooth manifold are the same! 
The theory of 3-manifolds also changed its view in the 80's. The theory of 3-manifolds was strongly influenced by the Poincaré conjecture but the progress was slow before 1957. In 1957, Papakyriakopoulos proved three technical theorems (the sphere and loop theorem as well Dehn's lemma) with tremendous impact on the whole 3-manifold theory. Next, Milnor proved that every compact 3-manifold can be split into an unique decomposition of prime manifolds (3-manifolds which are not splittable by using the connected sum). In 1979, Jaco, Shalen and Johannson found a finer decomposition of some prime manifolds by cutting them into pieces along embedded tori (JSJ decomposition). But the real breakthrough came from Thurston around 1980. His work was inspired by the construction of geometric structures (metric of constant curvature simulated by a homogeneous space) for the complement of knots in the 3sphere. Based on this examples, he conjectured that any compact 3-manifold can be cutted into pieces so that every piece admits a geometric structure (Geometrization conjecture). If this conjecture is true then Poincare's conjecture is also settled. So, the topological structure of 3-manifolds has a lot to do with its geometry! In the 80's, Hamilton developed the Ricci flow technique to prove this conjecture with very interesting results. But in 2002 and 2003, Perelman submitted three papers to arxiv.org in which he proposed a proof of the Geometrization conjecture. The arguments were checked by many mathematicians but no error was found. It seems that a 3-manifold is determined by a flow in the space of metrics.

\section{DIFFERENTIAL TOPOLOGY UNVEILS THE QUANTUM NATURE OF SPACETIME}

As mentioned above, differential topology is the mathematical theory of smooth manifolds including the (smooth) relations between submanifolds. In the first section, the basic assumptions of quantum gravity were discussed. The usage of a smooth 4-manifold as spacetime was not questioned. However the choice of the smoothness structure is not unique and I will discuss it now. As starting point consider a toy model to visualize the changes of the smoothness structure. Start with a torus (or doughnut) $T^{2}=S^{1} \times S^{1}$. Now one cuts the torus along one circle to obtain a cylinder, twist one end of the cylinder by $2 \pi$ and glue the two ends together. This process is called a Dehn twist and one obtains a torus again but now with a twist. But by the classification of closed surfaces, the usual and the twisted torus are diffeomorphic to each other. This fact is amazing but both tori are located in different components of the diffeomorphism group, i.e. in two different isotopy classes. In particular there is no coordinate transformation (i.e. a diffeomorphism connected to the identity) which transforms the twisted torus to the usual torus. This toy model shows the difference between coordinate transformations (diffeomorphisms connected to the identity) and global diffeomorphisms. A similar effect is the change of the smoothness structure (to a non-equivalent one) but it cannot be visualized in this easy way. As an example of this change I consider a compact 4-manifold $M$ (topologically complicated enough, i.e. a K3 surface or more) containing a special torus $T_{c}^{2}$ (so called c-embedded torus). Now cut out a neighborhood $D^{2} \times T_{c}^{2}$ of this torus (with boundary a 3-torus $T^{3}$ ) and glue in $\left(S^{3} \backslash\left(D^{2} \times K\right)\right) \times S^{1}$ (having also the boundary $\left.T^{3}\right)$ where $S^{3} \backslash\left(D^{2} \times K\right)$ denotes the complement of a knot $K$ in the 3 -sphere $S^{3}$. Then one obtains

$$
M_{K}=\left(M \backslash\left(D^{2} \times T_{c}^{2}\right)\right) \cup_{T^{3}}\left(\left(S^{3} \backslash\left(D^{2} \times K\right)\right) \times S^{1}\right)
$$

a new 4-manifold $M_{K}$ which is homeomorphic to $M$ (Fintushel-Stern knot surgery [16]). If the knot is non-trivial then $M_{K}$ is not diffeomorphic to $M$. One calls $M_{K}$ an exotic 4-manifold, a misleading term. Nothing is really exotic here. Nearly all smoothness structures on a 4-manifold are exotic.

Now consider the physically significant non-compact examples of exotic 4-manifolds like $\mathbb{R}^{4}$ and $S^{3} \times \mathbb{R}$. Start with $S^{3} \times \mathbb{R}$. This non-compact 4-manifold has the usual form used in GR. There is a global foliation along $\mathbb{R}$, i.e. $S^{3} \times\{t\}$ with $t \in \mathbb{R}$ are the (spatial) leafs. $S^{3} \times \mathbb{R}$ with this foliation is called the "standard $S^{3} \times \mathbb{R}$ ". I will denote an exotic version by $S^{3} \times{ }_{\theta} \mathbb{R}$. The construction of $S^{3} \times_{\theta} \mathbb{R}$ is rather complicated (see [17]). As a main ingredient one needs a homology 3 -sphere $\Sigma$ (i.e. a compact, closed 3-manifold with the homology groups of the 3-sphere) which does not bound a contractable 4-manifold (i.e. a 4-manifold which can be contracted to a point by a smooth homotopy). Interestingly, this homology 3-sphere $\Sigma$ is smoothly embedded in $S^{3} \times_{\theta} \mathbb{R}$ (as cross section, i.e. $\Sigma \times\{0\} \subset S^{3} \times{ }_{\theta} \mathbb{R}$ ). From the geometrical point of view, this 3-manifold is also very interesting. One can choose $\Sigma$ so that it admits a hyperbolic structure, i.e. a homogeneous metric of constant negative curvature. Hyperbolic 3-manifolds have a special property: Mostow rigidity [18]. Every diffeomorphism (especially every conformal transformation) of a hyperbolic 3-manifold is induced by an isometry. Therefore the volume and the curvature (or better the ChernSimons invariant) are topological invariants for hyperbolic 3-manifolds. In particular there are surfaces in hyperbolic 3-manifolds (incompressible surfaces) having a special (not divisible) volume. Then one obtains also a kind of quantized areas by purely topological methods.

What about the foliation of $S^{3} \times_{\theta} \mathbb{R}$ ? There is no foliation along $\mathbb{R}$ but there is a codimension-one foliation of the 3-sphere $S^{3}$ (see [19] for the construction). So, $S^{3} \times_{\theta} \mathbb{R}$ is foliated along $S^{3}$ and the leafs are $S_{i} \times \mathbb{R}$ with the surfaces $\left\{S_{i}\right\}_{i \in I} \subset S^{3}$. But what happens with the 3 -spheres in $S^{3} \times_{\theta} \mathbb{R}$ ? There is no smoothly embedded $S^{3}$ in $S^{3} \times{ }_{\theta} \mathbb{R}$ (otherwise it would have the standard smoothness structure). But there is a wildly embedded $S^{3}$ ! Let $i: K \rightarrow M$ 
be an embedding of $K$ (with $\operatorname{dim} K<\operatorname{dim} M$ ). One calls the embedding $i$ wild if $i(K)$ is not a finite polyhedron (or $i(K)$ is not triangulated by a finite number of simplices). In [10], we considered wildly embedded submanifolds as models of quantum D-branes. The prominent example of a wildly embedded submanifold is Alexanders horned sphere. Wild embedded submanifolds are fractals in a generalized manner. Now I will argue in the following that this wild embedding is a geometric model for a quantum state.

As discussed in the first section, all approaches of quantum gravity have problems with the construction of the state space. If I assume that the spacetime has the right properties for a spacetime picture of quantum gravity then the quantum state must be part of the spacetime or must be geometrically realized in the spacetime. Consider (as in geometrodynamics) a 3 -sphere $S^{3}$ with metric $g$. This metric (as state of GR) is modeled on $S^{3}$ at every 3 -dimensional subspace. If $g$ is a metric of a homogeneous space then one can choose a small coordinate patch. But if $g$ is inhomogeneous then one can use a diffeomorphism to "concentrate" the inhomogeneity at a chart. Now one combines these infinite charts (I consider only metrics up to diffeomorphisms) into a 3-sphere but without destroying the infinite charts by a diffeomorphism. Wild embeddings are the right structure for this idea. A wild embedding cannot be undone by a diffeomorphism of the embedding space. Secondly, this wild embedding of a 3-sphere into $S^{3} \times_{\theta} \mathbb{R}$ is determined by its complement. So, if one understands the complement of the wild embedding then one understands the wild embedding itself. Interestingly, one can construct an operator algebra and a Hilbert space from a wild embedding (see Appendix A, below). This operator algebra can be also obtained from the foliation (by using the noncommutative geometry approach), see $[11,20]$. It is the hyperfinite factor $I I I_{1}$ von Neumann algebra having the structure of the local algebras in a relativistic QFT with one vacuum vector. Then one obtains a backgroundindependent approach but how does the classical spacetime appears? A wild embedding is defined by the infinite polyhedron. So, if I reduces the wild embedding to a finite polyhedron by contracting the smaller parts to zero then one obtains a finite polyhedron. But by definition, a finite polyhedron is not a wild embedding (or comes from a wild embedding). In [10], the process was discussed for quantum D-branes. Especially, the classical action of a D-brane was obtained. In summary, we obtained a state space as operator algebra of the wild embedding induced by exotic smoothness structures. The state space is

1. background-independent (diffeomorphism invariant)

2. with countable infinite basis (discrete structure)

3. and contains the classical spacetime as limit.

Of course, the whole approach is very theoretical up to now. For instance I do not start with a concrete action or list of fields. But sometimes, things went better than expected. In [13], we considered the Fintushel-Stern knot surgery above to obtain (1), the exotic $M_{K}$. If one started with the Einstein-Hilbert action on $M$ then we obtained the combined Einstein-Hilbert-Dirac-Yang-Mills system. The knot complement is directly related to the fermions whereas the bosons appear as torus bundles. A lot of work has to be done but it is a beginning.

\section{CONCLUSION}

I have presented a certain number of ideas and results:

1. There is a freedom in the definition of the spacetime coming from the choice of the smoothness structure.

2. There are an infinity of exotic smoothness structures to choose from. For example consider the foliation of an exotic spacetime like $S^{3} \times_{\theta} \mathbb{R}$ can be very complicated.

3. For the usual foliation $S^{3} \times\{t\}$ with $t \in \mathbb{R}$ of $S^{3} \times_{\theta} \mathbb{R}$ the 3 -sphere must be a wildly embedded submanifold (represented by an infinite polyhedron).

4. A quantum state can be defined on the spacetime as wild embedding.

5. A glimpse of an action to obtain a full QFT and quantum gravity is also obtained.

Before concluding, I must add that the views expressed are only partly original. I have partially drawn from the ideas of Carl H. Brans, Jerzy Król and Helge Rosé. 


\section{Appendix A: $C^{*}-$ algebras associated to wild embeddings}

Let $I: K^{n} \rightarrow \mathbb{R}^{n+k}$ be a wild embedding of codimension $k$ with $k=0,1,2$. In the following we assume that the complement $\mathbb{R}^{n+k} \backslash I\left(K^{n}\right)$ is non-trivial, i.e. $\pi_{1}\left(\mathbb{R}^{n+k} \backslash I\left(K^{n}\right)\right)=\pi \neq 1$. Now one defines the $C^{*}$-algebra $C^{*}(\mathcal{G}, \pi)$ associated to the complement $\mathcal{G}=\mathbb{R}^{n+k} \backslash I\left(K^{n}\right)$ with group $\pi=\pi_{1}(\mathcal{G})$. If $\pi$ is non-trivial then this group is not finitely generated. The construction of wild embeddings is given by an infinite construction[24] (see Antoine's necklace or Alexanders horned sphere). From an abstract point of view, we have a decomposition of $\mathcal{G}$ by an infinite union

$$
\mathcal{G}=\bigcup_{i=0}^{\infty} C_{i}
$$

of "level sets" $C_{i}$. Then every element $\gamma \in \pi$ lies (up to homotopy) in a finite union of levels.

The basic elements of the $C^{*}$-algebra $C^{*}(\mathcal{G}, \pi)$ are smooth half-densities with compact supports on $\mathcal{G}, f \in$ $C_{c}^{\infty}\left(\mathcal{G}, \Omega^{1 / 2}\right)$, where $\Omega_{\gamma}^{1 / 2}$ for $\gamma \in \pi$ is the one-dimensional complex vector space of maps from the exterior power $\Lambda^{2} L$, of the union of levels $L$ representing $\gamma$, to $\mathbb{C}$ such that

$$
\rho(\lambda \nu)=|\lambda|^{1 / 2} \rho(\nu) \quad \forall \nu \in \Lambda^{2} L, \lambda \in \mathbb{R} .
$$

For $f, g \in C_{c}^{\infty}\left(\mathcal{G}, \Omega^{1 / 2}\right)$, the convolution product $f * g$ is given by the equality

$$
(f * g)(\gamma)=\int_{\gamma_{1} \circ \gamma_{2}=\gamma} f\left(\gamma_{1}\right) g\left(\gamma_{2}\right)
$$

with the group operation $\gamma_{1} \circ \gamma_{2}$ in $\pi$. Then we define via $f^{*}(\gamma)=\overline{f\left(\gamma^{-1}\right)}$ a $*$ operation making $C_{c}^{\infty}\left(\mathcal{G}, \Omega^{1 / 2}\right)$ into a $*$ algebra. Each level set $C_{i}$ consists of simple pieces (for instance tubes in case of the Alexanders horned sphere) denoted by $T$. For these pieces, one has a natural representation of $C_{c}^{\infty}\left(\mathcal{G}, \Omega^{1 / 2}\right)$ on the $L^{2}$ space over $T$. Then one defines the representation

$$
\left(\pi_{x}(f) \xi\right)(\gamma)=\int_{\gamma_{1} \circ \gamma_{2}=\gamma} f\left(\gamma_{1}\right) \xi\left(\gamma_{2}\right) \quad \forall \xi \in L^{2}(T), \forall x \in \gamma .
$$

The completion of $C_{c}^{\infty}\left(\mathcal{G}, \Omega^{1 / 2}\right)$ with respect to the norm

$$
\|f\|=\sup _{x \in \mathcal{G}}\left\|\pi_{x}(f)\right\|
$$

makes it into a $C^{*}$ algebra $C_{c}^{\infty}(\mathcal{G}, \pi)$. The $C^{*}$-algebra $C_{c}^{\infty}(K, I)$ associated to the wild embedding $I$ is defined to be $C_{c}^{\infty}(K, j)=C_{c}^{\infty}(\mathcal{G}, \pi)$. The GNS representation of this algebra is called the state space. 


\section{References}

[1] T. Asselmeyer, Generation of source terms in general relativity by differential structures, Class. Quant. Grav. 14 (1996) $749-758$.

[2] T. Asselmeyer-Maluga, C. Brans, Cosmological anomalies and exotic smoothness structures, Gen. Rel. Grav. 34 (2002) 597-607.

[3] T. Asselmeyer-Maluga, H. Rosé, Dark Energy and 3-Manifold Topology, Act. Phys. Pol. 38 (2007) 3633-3639.

[4] T. Asselmeyer-Maluga, C. Brans, Exotic Smoothness and Physics, WorldScientific Publ., Singapore, 2007.

[5] T. Asselmeyer-Maluga, Exotic Smoothness and Quantum Gravity, Class. Quantum Grav. 27 (2010) 165002, arXiv:1003.5506v1 [gr-qc].

[6] T. Asselmeyer-Maluga, J. Król, Small exotic smooth $R^{4}$ and string theory, in: International Congress of Mathematicians ICM 2010 Short Communications Abstracts Book, Ed. R. Bathia, Hindustan Book Agency, 400, 2010.

[7] T. Asselmeyer-Maluga, J. Król, Exotic Smooth $R^{4}$ and certain configurations of NS and D branes in string theory, Int. J. Mod. Phys. A 26 (2011) 1375 - 1388, arXiv: 1101.3169.

[8] T. Asselmeyer-Maluga, J. Król, Constructing a Quantum Field Theory from Spacetime, arXiv:1107.3458, 2011.

[9] T. Asselmeyer-Maluga, J. Krol, Quantum D-Branes and Exotic Smooth $\mathbb{R}^{4}$, Int. J. Geom. Methods in Modern Physics 9 (2012) 1250022, arXiv:1102.3274.

[10] T. Asselmeyer-Maluga, J. Król, Topological Quantum D-Branes and Wild Embeddings from Exotic Smooth $R^{4}$, Int. J. Mod. Phys. A26 (2011) 3421 - 3437, arXiv:1105.1557.

[11] T. Asselmeyer-Maluga, R. Mader, Exotic $R^{4}$ and Quantum Field Theory, in: C. B. E. Al. (Ed.), 7th International Conference on Quantum Theory and Symmetries (QTS7), IOP Publishing, Bristol, UK, 012011, arXiv:1112.4885, doi:10.1088/1742-6596/343/1/012011, 2012.

[12] T. Asselmeyer-Maluga, J. Król, On Topological Restrictions of the Spacetime in Cosmology, Mod. Phys. Lett. A 27 (2012) 1250135, arXiv:1206.4796.

[13] T. Asselmeyer-Maluga, H. Rosé, On the Geometrization of Matter by Exotic Smoothness, Gen. Rel. Grav. DOI: 10.1007/s10714-012-1419-3, arXiv:1006.2230.

[14] A. Bernal, M. Saánchez, Smoothness of Time Functions and the Metric Splitting of Globally Hyperbolic Space Times, Commun. Math. Phys. 257 (2005) 43-50, arXiv:gr-qc/0401112.

[15] A. Bernal, M. Saánchez, Globally Hyperbolic Spacetimes Can Be Defined as "causal" Instead of "strongly Causal", Class. Quant. Grav. 24 (2007) 745-750, arXiv:gr-qc/0611138.

[16] R. Fintushel, R. Stern, Knots, links, and 4-manifolds, Inv. Math 134 (1998) 363-400, (dg-ga/9612014).

[17] M. Freedman, A Fake $S^{3} \times R$, Ann. of Math. 110 (1979) 177-201.

[18] G. Mostow, Quasi-conformal mappings in $n$-space and the rigidity of hyperbolic space forms, Publ. Math. IHÉS 34 (1968) 53-104.

[19] T. Asselmeyer-Maluga, J. Król, Abelian Gerbes, Generalized Geometries and Exotic $R^{4}$, arXiv: 0904.1276, subm. to J. Math. Phys., 2009.

[20] T. Asselmeyer-Maluga, J. Król, Exotic smooth $\mathbb{R}^{4}$, noncommutative algebras and quantization, arXiv: $1001.0882,2010$.

[21] C. Rovelli, Quantum Gravity, Cambridge Monographs on Mathematical Physics, Cambridge University Press, Cambridge, www.cpt.univ-mrs.fr/ rovelli/book.pdf, 2004.

[22] There are many books about quantum gravity, for instance [21], and the original papers which I omit to cite.

[23] Current experiments at the LHC do not give any sign for extra dimensions (see the Particle Data Group).

[24] This infinite construction is necessary to obtain an infinite polyhedron, the defining property of a wild embedding. 